\title{
The Formation of the First Low-Mass Stars From
}

\section{Gas With Low Carbon and Oxygen Abundances}

Volker Bromm and Abraham Loeb

Astronomy Department, Harvard University, 60 Garden Street, Cambridge, MA 02138, USA

Appeared in Nature, 425, 812-814 (2003)

The first stars in the Universe are predicted to have been much more massive than the $\operatorname{Sun}^{1-3}$. Gravitational condensation accompanied by cooling of the primordial gas due to molecular hydrogen, yields a minimum fragmentation scale of a few hundred solar masses. Numerical simulations indicate that once a gas clump acquires this mass, it undergoes a slow, quasi-hydrostatic contraction without further fragmentation ${ }^{1,2}$. Here we show that as soon as the primordial gas - left over from the Big Bang - is enriched by supernovae to a carbon or oxygen abundance as small as $\sim 0.01-0.1 \%$ of that found in the Sun, cooling by singly-ionized carbon or neutral oxygen can lead to the formation of low-mass stars. This mechanism naturally accommodates the discovery ${ }^{4}$ of solar mass stars with unusually low $\left(10^{-5.3}\right.$ of the solar value) iron abundance but with a high $\left(10^{-1.3}\right.$ solar $)$ carbon abundance. The minimum stellar mass at early epochs is partially regulated by the temperature of the cosmic microwave background. The derived critical abundances can be used to identify those metal-poor stars in our Milky Way galaxy with elemental patterns imprinted by the first supernovae.

The microphysics of cooling due to molecular hydrogen $\left(\mathrm{H}_{2}\right)$ within the first gas clouds to condense in the Universe, introduces a characteristic temperature $\left(T_{c} \sim 100-200 \mathrm{~K}\right)$ and hydrogen density $\left(n_{c} \sim 10^{4} \mathrm{~cm}^{-3}\right)$ into the formation process of the first $\operatorname{stars}^{1,2}$. The 
temperature floor is set by the energy separation of the lowest-lying rotational levels of $\mathrm{H}_{2}$, and the characteristic density leads to the thermalization of these levels, at which point cooling becomes less efficient. The minimum mass scale for fragmentation is approximately given by the Jeans mass, $M_{J} \simeq 400 M_{\odot}(T / 200 \mathrm{~K})^{3 / 2}\left(n / 10^{4} \mathrm{~cm}^{-3}\right)^{-1 / 2}$. The final mass of a star is determined by the accretion process onto the nascent protostellar core, resulting in $M_{*} \simeq$ $\alpha M_{J}$ with $\alpha \lesssim 0.5^{5,6}$. In contrast to the formation mode of massive stars (population III) at high redshifts, fragmentation is observed to favor stars below a solar mass (population I and II) in the present-day Universe. The transition between these fundamental modes is expected to be mainly driven by the progressive enrichment of the cosmic gas with heavy elements (or 'metals'), which enable the gas to cool to lower temperatures. The concept of a 'critical metallicity', $Z_{\text {crit }}$, has been used $^{7-11}$ to characterize the transition between population III and population II formation modes (where $Z$ denotes the mass fraction contributed by all heavy elements). Previous studies ${ }^{7,8,11}$ have only constrained this important parameter to within a few orders of magnitude, $Z_{\text {crit }} \sim 10^{-6}-10^{-3} Z_{\odot}$, under the implicit assumption of solar relative abundances of metals. This assumption is likely to be violated by the metal yields of the first supernovae (SNe) at high-redshifts, for which strong deviations from solar abundance ratios are predicted ${ }^{12-17}$. The cooling rate of the metals depends on their ionization state, which in turn is controlled by the ionizing backgrounds (UV and X-ray photons or cosmic rays) that were not fully considered in earlier studies.

Here we show that the transition between the above star formation modes is driven primarily by fine-structure line cooling of singly-ionized carbon or neutral atomic oxygen. We refine earlier estimates of $Z_{\text {crit }}$ which did not explicitly distinguish between different coolants, by introducing separate critical abundances for carbon and oxygen, $[\mathrm{C} / \mathrm{H}]_{\text {crit }}$ and $[\mathrm{O} / \mathrm{H}]_{\text {crit }}$, respectively, where $[\mathrm{A} / \mathrm{H}]=\log _{10}\left(N_{\mathrm{A}} / N_{\mathrm{H}}\right)-\log _{10}\left(N_{\mathrm{A}} / N_{\mathrm{H}}\right)_{\odot}$, and a subscript ' $\odot$ ' denotes solar values. Why are $C$ and $O$ identified as the key species responsible for the global shift in the star formation mode? We find that under the temperature and density 
conditions that characterize population III star formation, the most important coolants are O I and C II whose fine-structure lines dominate over all other metal transitions ${ }^{18}$. Cooling due to molecules becomes important only at lower temperatures, and cooling due to dust grains only at higher densities ${ }^{18,7,11}$.

The conditions that terminate the predominance of massive, population III stars, have dramatic implications for the reionization history of the Universe. Massive stars are much more efficient at producing ionizing radiation than low mass stars, due to their high surface temperature $\left(\sim 10^{5} \mathrm{~K}\right)$. In particular, metal-free stars with a mass $\gtrsim 100 M_{\odot}$ produce $\sim$ $5 \times 10^{4}$ hydrogen ionizing photons per baryon incorporated into them, a yield higher by an order of magnitude than obtained for a present-day mass function of stars ${ }^{19,20}$. Massive stars can therefore reionize the universe early ${ }^{21-23}$ at $z \sim 17$, as required by the recent detection ${ }^{24}$ of large scale polarization anisotropies in the cosmic microwave background (CMB). A strong UV flux just below the Lyman limit $(h \nu<13.6 \mathrm{eV})$ is predicted $^{25}$ to be emitted by the same stars that are responsible for the reionization of the IGM. This soft UV radiation can penetrate the neutral IGM and ionize any trace amount of neutral carbon due to its low firstionization potential of $11.26 \mathrm{eV}$. Since carbon is highly underabundant, the UV background can ionize carbon throughout the universe well before hydrogen reionization. Oxygen, on the other hand, will be predominantly neutral prior to reionization since its ionization potential is $13.62 \mathrm{eV}$. Cooling is mediated by excitations due to collisions of the respective metal with free electrons or hydrogen atoms. At the low fractional abundances of electrons, $x_{e} \lesssim 10^{-4}$, expected in the neutral (rapidly recombining) gas at $z \sim 15$, collisions with hydrogen atoms dominate. Even if the gas had initially been ionized to a much higher degree due to the impact of the supernova ejecta, it would have recombined to the level stated above within its free-fall time $\left[t_{\mathrm{ff}} \sim 5 \times 10^{5}\left(\mathrm{n} / 10^{4} \mathrm{~cm}^{-3}\right)^{-1 / 2} \mathrm{yr}\right]$. This renders our analysis independent of the very uncertain hydrogen-ionizing backgrounds at high redshifts (cosmic rays or soft X-rays). 
We now derive the critical $\mathrm{C}$ and $\mathrm{O}$ abundances. Our starting point is the characteristic state reached in primordial gas that cools only through molecular hydrogen. For the gas to fragment further (instead of following a slow contraction to a single massive star ${ }^{1,2}$ ), additional cooling due to C II or O I is required. Fragmentation requires that the radiative cooling rate be higher than the free-fall compressional heating rate. We find the critical metal abundances by equating the two rates: $\Lambda_{\mathrm{CII}, \mathrm{O}}(n, T) \simeq 1.5 n k_{\mathrm{B}} T / t_{\mathrm{ff}}$, where $k_{\mathrm{B}}$ is Boltzmann's constant. In evaluating this threshold condition, we solve the respective rate equations for C II (a two-level system) and O I (a three-level system), including all possible radiative and collisional transitions ${ }^{18}$. We assume that the emission of the fine-structure lines proceeds under optically thin conditions, as appropriate for the low atomic abundances considered here $^{18}$. We find the critical $\mathrm{C}$ and $\mathrm{O}$ abundances by setting $n \sim n_{c}$ and $T \sim T_{c}$, and estimate the uncertainties by considering a plausible range near these values $(100 \mathrm{~K} \lesssim T \lesssim 200 \mathrm{~K})$.

In Fig. 1, we show the threshold $\mathrm{C}$ and $\mathrm{O}$ abundances that enable continued fragmentation for various temperature and density values. It is convenient to plot these quantities as functions of the Jeans mass $\left(\propto T^{3 / 2} n^{-1 / 2}\right)$. We find critical abundance values of $[\mathrm{C} / \mathrm{H}]_{\text {crit }} \simeq-3.5 \pm 0.1$ and $[\mathrm{O} / \mathrm{H}]_{\text {crit }} \simeq-3.05 \pm 0.2$. Strictly speaking, these threshold levels are the required abundances in the gas phase. If a fraction of these elements were depleted onto dust grains, then the total $\mathrm{C}$ and $\mathrm{O}$ elemental abundances would have to be correspondingly higher. Considering the large current uncertainties in modelling the properties and formation channels of dust at low metal abundances ${ }^{11}$, we assume the limiting case of zero $\mathrm{C}$ and $\mathrm{O}$ depletion onto dust when comparing our theoretical predictions with observed stellar abundances.

We stress that even if sufficient $\mathrm{C}$ or $\mathrm{O}$ atoms are present to further cool the gas, there will be a minimum attainable temperature ${ }^{26,6}$ that is set by the interaction of the atoms with the thermal CMB: $T_{\mathrm{CMB}}=2.7 \mathrm{~K}(1+z)$. At $z \simeq 15$, this results in a minimum fragment 
mass of $M_{*} \sim 20 M_{\odot}\left(n_{f} / 10^{4} \mathrm{~cm}^{-3}\right)^{-1 / 2}$, where $n_{f}>10^{4} \mathrm{~cm}^{-3}$ is the density at which opacity prevents further fragmentation ${ }^{27}$. It is possible that the transition from the high-mass to the low-mass star formation mode was modulated by the CMB temperature and was therefore gradual ${ }^{6}$, involving intermediate-mass ('population II.5') $\operatorname{stars}^{10}$ at intermediate redshifts. This transitional population can give rise to the faint SNe that have been proposed to explain the observed abundance patterns in metal-poor stars ${ }^{15,17}$. These solar-mass stars themselves should have formed out of metal-poor gas at relatively late times when the CMB temperature was sufficiently low.

In relating our results to theoretical nucleosynthetic yields, one has to address the degree of mixing, or dilution, that the SN ejecta experience. SNe at high redshifts occur in dark matter halos with shallow potential wells, so that the resulting blast wave is predicted ${ }^{28}$ to escape into the expanding IGM. The dilution mass can then be estimated as $M_{\text {dil }} \simeq$ $2 E_{\mathrm{SN}} / v^{2}$, where $E_{\mathrm{SN}}$ is the explosion kinetic energy, and $v=H(z) r$ is the Hubble velocity at a distance $r$. Using standard cosmological parameters and ignoring radiative losses ${ }^{28}$, we get $M_{\mathrm{dil}} \simeq 10^{7} M_{\odot}\left(E_{\mathrm{SN}} / 10^{53} \mathrm{ergs}\right)^{3 / 5}[(1+z) / 16]^{-3 / 5}$. In the expected mass range of the pair-instability supernovae ${ }^{12}(\mathrm{PISNe})$ and the corresponding explosion energies $E_{\mathrm{SN}} \simeq$ $10^{51}-10^{53}$ ergs, one finds dilution masses that are more than two orders of magnitude larger than typical values for type II SNe in nearby galaxies ${ }^{14}$. However, depending on the exact values of the ejected mass of $\mathrm{C}$ and $\mathrm{O}$ and the respective explosion energies, a single SN event could already lead to $[\mathrm{C} / \mathrm{H}]$ and $[\mathrm{O} / \mathrm{H}]$ ratios that 'overshoot' the critical abundances. For example, the abundances resulting from the explosion and subsequent mixing of a PISN at the low-mass end ${ }^{12}$, are $[\mathrm{C} / \mathrm{H}] \simeq-2.9$ and $[\mathrm{O} / \mathrm{H}] \simeq-2.4$, respectively, roughly 0.5 dex above the critical levels. Nevertheless, a single PISN at the high-mass end would produce final, mixed abundances still below critical. Since the dilution mass scales as the number of simultaneous SNe to the $3 / 5$ power while the ejecta mass scales linearly with this number, multiple simultaneous SNe within the same galaxy would be less effective 
at diluting their metal abundances into the expanding IGM. But further dilution may occur during the hierarchical assembly of galaxies, as infall may add metal-free gas from the IGM to the enriched interstellar medium.

In Fig. 2 we compare our theoretical thresholds to the observed $\mathrm{C}$ and $\mathrm{O}$ abundances in metal-poor dwarf ${ }^{29}$ and giant ${ }^{30}$ stars in the halo of our Galaxy. As can be seen, all data points lie above the critical $\mathrm{O}$ abundance but a few cases lie below the critical $\mathrm{C}$ threshold. All of these low mass stars are consistent with our model since the corresponding O abundances lie above the predicted threshold. The sub-critical $[\mathrm{C} / \mathrm{H}]$ abundances could have either originated in the progenitor cloud or from the mixing of CNO-processed material (with carbon converted into nitrogen) into the stellar atmosphere during the red giant phase. To guard against this a posteriori processing of C, dwarf stars should be preferred as they provide a more reliable record of the primordial abundance pattern that was present at birth. However, since dwarfs are fainter, another strategy would be to focus on giants which show evidence for weak mixing (e.g. in terms of high ${ }^{12} \mathrm{C} /{ }^{13} \mathrm{C}$ isotope ratio or low ${ }^{14} \mathrm{~N}$ abundance). The current data on dwarf stars (filled symbols in Fig. 2) does not yet reach C and $\mathrm{O}$ abundances that are sufficiently low to probe the theoretical cooling thresholds. Note also that the extremely iron-poor star HE0107-5240 has C and O abundances that both lie above the respective critical levels (Bessell, M., et al., in preparation). The formation of this low mass star $\left(\sim 0.8 M_{\odot}\right)$ is therefore consistent with the theoretical framework considered here $^{11,17}$.

Our model suggests a powerful new probe of the first SNe to have exploded in the Universe. By selecting an unbiased new sample of Galactic halo stars with $\mathrm{C}$ or $\mathrm{O}$ abundances close to the theoretical critical values, it should be possible to sample individual SN events originating in the earliest epochs of cosmic star formation. The value of $[\mathrm{C} / \mathrm{Fe}]$ would be indicative of the particular $\mathrm{SN}$ type. For example, the very high value of $[\mathrm{C} / \mathrm{Fe}] \simeq+4$ 
observed $^{4}$ in HE0107-5240, can be explained by a core collapse SN which leaves behind a black hole after fallback of most of the outer stellar envelope ${ }^{17}$. Modern surveys select metal-poor stars based on their weak or absent Ca II K-lines. Our model suggests that a better strategy to select truly 'second generation' stars is to look for stars with the lowest abundances of carbon and oxygen. 


\section{REFERENCES}

${ }^{1}$ Abel, T., Bryan, G. \& Norman, M. The formation of the first star in the universe. Science 295, 93-98 (2002).

${ }^{2}$ Bromm, V., Coppi, P.S. \& Larson, R.B. The formation of the first stars. I. The primordial starforming cloud. Astrophys. J. 564, 23-51 (2002).

${ }^{3}$ Nakamura, F. \& Umemura, M. The stellar initial mass function in primordial galaxies. Astrophys. J. 569, 549-557 (2002).

${ }^{4}$ Christlieb, N. et al. A stellar relic from the early Milky Way. Nature 419, 904-906 (2002).

${ }^{5}$ McKee, C. F. \& Tan, J. C. Massive star formation in 100,000 years from turbulent and pressurized molecular clouds. Nature 416, 59-61 (2002).

${ }^{6}$ Clarke, C. J. \& Bromm, V. The characteristic stellar mass as a function of redshift. Mon. Not. R. Astron. Soc. 343, 1224-1230 (2003).

${ }^{7}$ Omukai, K. Protostellar collapse with various metallicities. Astrophys. J. 534, 809-824 (2000).

${ }^{8}$ Bromm, V., Ferrara, A., Coppi, P.S. \& Larson, R.B. The fragmentation of pre-enriched primordial objects. Mon. Not. R. Astron. Soc. 328, 969-976 (2001).

${ }^{9}$ Schneider, R., Ferrara, A., Natarajan, P. \& Omukai, K. First stars, very massive black holes, and metals. Astrophys. J. 571, 30-39 (2002).

${ }^{10}$ Mackey, J., Bromm, V. \& Hernquist, L. Three epochs of star formation in the high-redshift universe. Astrophys. J. 586, 1-11 (2003).

${ }^{11}$ Schneider, R., Ferrara, A., Salvaterra, R., Omukai, K. \& Bromm, V. Low-mass relics of early star formation. Nature 422, 869-871 (2003).

${ }^{12}$ Heger, A. \& Woosley, S. E. The nucleosynthetic signature of population III. Astrophys. J. 567, 532-543 (2002).

${ }^{13}$ Qian, Y.-Z., Sargent, W. L. W. \& Wasserburg, G. J. The prompt inventory from very massive stars and elemental abundances in Ly $\alpha$ systems. Astrophys. J. 569, L61-L64 (2002).

${ }^{14}$ Qian, Y.-Z. \& Wasserburg, G. J. Determination of nucleosynthetic yields of supernovae and very massive stars from abundances in metal-poor stars. Astrophys. J. 567, 515-531 (2002). 
${ }^{15}$ Umeda, H. \& Nomoto, K. Nucleosynthesis of zinc and iron peak elements in population III type II supernovae. Astrophys. J. 565, 385-404 (2002).

${ }^{16}$ Sneden, C. \& Cowan, J. J. Genesis of the heaviest elements in the Milky Way Galaxy. Science 299, 70-75 (2003).

${ }^{17}$ Umeda, H. \& Nomoto, K. First-generation black-hole-forming supernovae and the metal abundance pattern of a very iron-poor star. Nature 422, 871-873 (2003).

${ }^{18}$ Hollenbach, D. \& McKee, C. F. Molecule formation and infrared emission in fast interstellar shocks. Astrophys. J. 342, 306-336 (1989).

${ }^{19}$ Tumlinson, J. \& Shull, J. M. Zero-metallicity stars and the effects of the first stars on reionization. Astrophys. J. 528, L65-L68 (2000).

${ }^{20}$ Bromm, V., Kudritzki, R.P. \& Loeb, A. Generic spectrum and ionization efficiency of a heavy initial mass function for the first stars. Astrophys. J. 552, 464-472 (2001).

21 Cen, R. The implications of Wilkinson Microwave Anisotropy Probe observations for population III star formation processes. Astrophys. J. 591, L5-L8 (2003).

22 Sokasian, A., Yoshida, N., Abel, T., Hernquist, L. \& Springel V. Cosmic reionisation by stellar sources: Population III stars. Mon. Not. R. Astron. Soc. (submitted); preprint available at 〈http://xxx.lanl.gov/astro-ph/0302213〉(2003).

${ }^{23}$ Wyithe, J. S. B. \& Loeb, A. Was the universe reionized by massive metal-free stars? Astrophys. J. 588, L69-L72 (2003).

${ }^{24}$ Kogut, A., et al. Wilkinson Microwave Anisotropy Probe WMAP first year observations: TE polarization. Astrophys. J. (in the press); preprint available at $\langle$ http://xxx.lanl.gov/astro$\mathrm{ph} / 0302213\rangle(2003)$.

${ }^{25}$ Bromm, V. \& Loeb, A. Formation of the first supermassive black holes. Astrophys. J. (in the press); preprint available at 〈http://xxx.lanl.gov/astro-ph/0212400〉(2003).

${ }^{26}$ Larson, R.B. Early star formation and the evolution of the stellar initial mass function in galaxies. Mon. Not. R. Astron. Soc. 301, 569-581 (1998).

${ }^{27}$ Rees, M. J. Opacity-limited hierarchical fragmentation and the masses of protostars. Mon. Not. R. Astron. Soc. 176, 483-486 (1976). 
${ }^{28}$ Bromm, V., Yoshida, N. \& Hernquist, L. The first supernova explosions in the universe. Astrophys. J. (submitted); preprint available at 〈http://xxx.lanl.gov/astro-ph/0305333〉(2003).

29 Akerman, C.J., Carigi, L., Nissen, P.E., Pettini, M. \& Asplund, M. The evolution of the C/O ratio in metal-poor halo stars. Astron. Astrophys. (2003) (submitted) (2003).

30 Cayrel, R. et al. Abundance patterns and supernova yields in the early Galaxy from C to Zn. Astron. Astrophys. (2003) (submitted) (2003).

Acknowledgments The authors thank Rennan Barkana and particularly Tim Beers for helpful discussions, and are grateful to Tim Beers and Max Pettini for making their data available prior to publication. This work was supported in part by NSF, NASA, and the Guggenheim foundation (for A.L.).

Correspondence and requests for materials should be addressed to V.B. (email: vbromm@cfa.harvard.edu) or A.L. (email: loeb@cfa.harvard.edu). 
Fig. 1.- Required carbon and oxygen abundances (relative to solar values) for cooling a clump of metal-poor gas faster than its free-fall (compressional heating) time. The minimum abundances are shown as functions of the initial Jeans mass of the clump (with higher abundances allowing the clump to cool and further fragment). The upper panel shows the case of singly ionized carbon, and the lower panel refers to neutral oxygen. The curves are labeled by the hydrogen number density in units of $\mathrm{cm}^{-3}$, with the solid curve marking the characteristic density in pre-stellar clumps of metal-poor gas, $n_{c} \sim 10^{4} \mathrm{~cm}^{-3}$. Heavy filled dots indicate particular temperature values. The critical abundance is delineated by the horizontal dashed lines, with a heavy line indicating the central value and the adjacent light lines bracketing the uncertainty within the characteristic temperature range, $T_{c} \sim 100-$ $200 \mathrm{~K}$, and density, $\sim 10^{4} \mathrm{~cm}^{-3}$, of pre-stellar clumps. The figure indicates the minimum metal abundance at which fragmentation into low-mass stars starts, but it does not provide information about the minimum mass of the fragments at the end of the cooling process. 


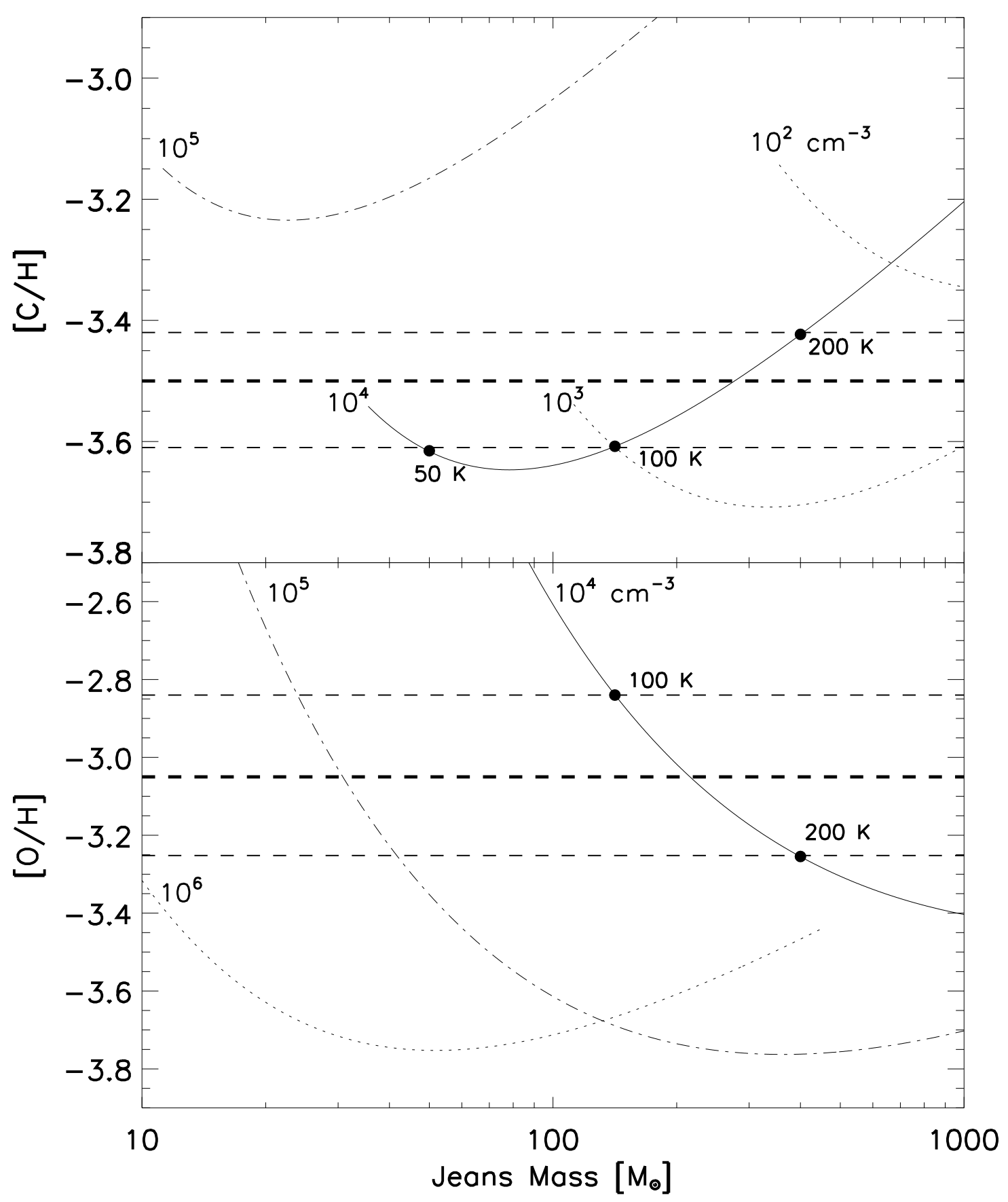


Fig. 2.- Observed abundances in low-metallicity Galactic halo stars. For both carbon (upper panel) and oxygen (lower panel), filled symbols correspond to samples of dwarf and subgiant stars $^{29}$, and open squares to a sample of giant stars ${ }^{30}$. Both data sets were obtained at a high signal-to-noise ratio using the UVES spectrograph on the VLT. The dashed lines indicate the predicted level of critical carbon and oxygen abundances from Fig. 1. According to our theoretical model, all low-mass dwarf stars ought to lie above the critical threshold level of at least one of the two elements. Indeed all stars in the existing data sets satisfy this condition. We highlight the location of the extremely iron-poor giant star HE0107-5240 (marked by $\mathbf{x}$ ), whose carbon and oxygen abundances are both above the critical levels ${ }^{4}$. 


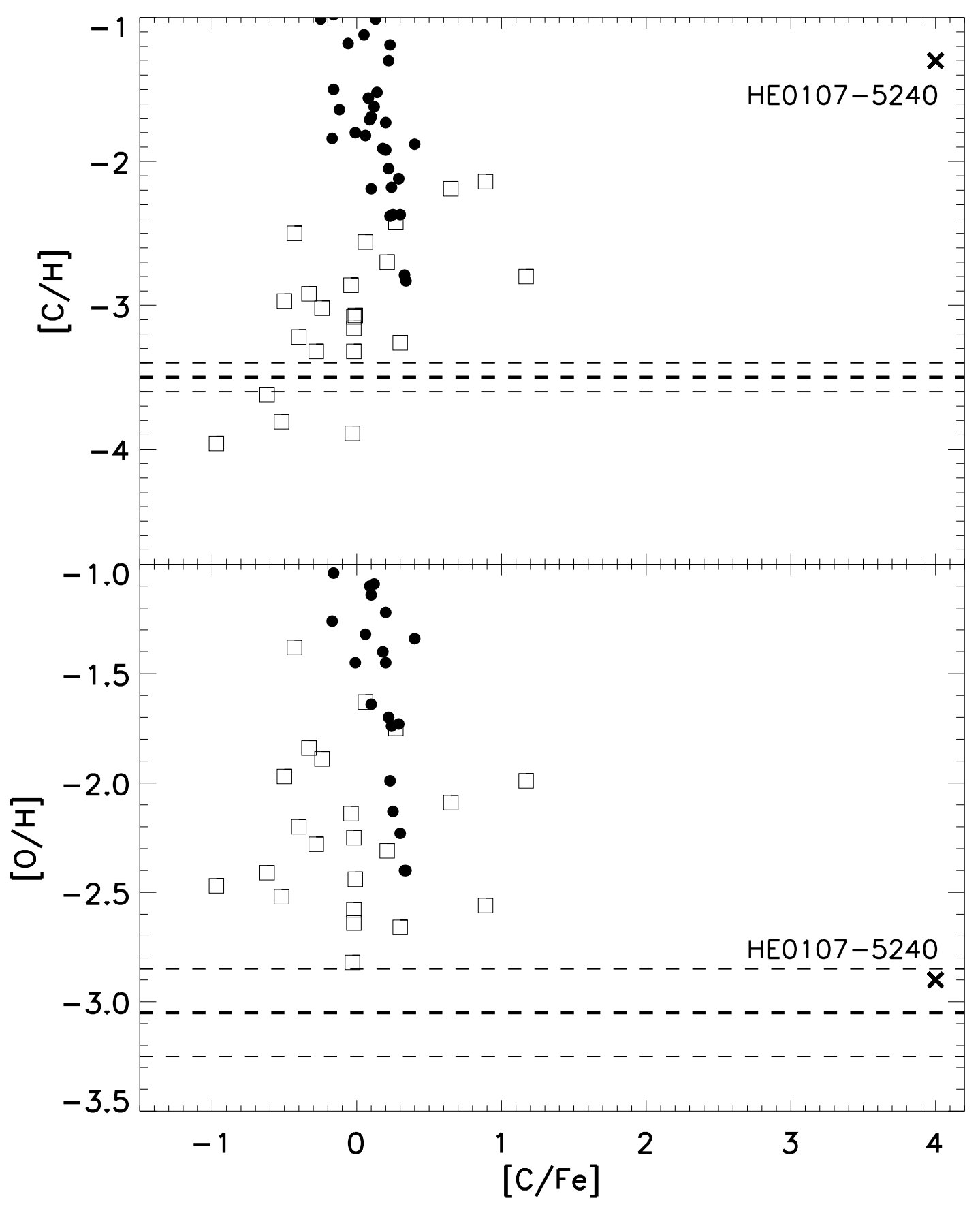

